\title{
BMJ Open Early PARacetamol (EPAR) trial: a study protocol for a randomised controlled trial of early paracetamol to promote closure of the ductus arteriosus in preterm infants
}

Tim Schindler, ${ }^{1,2}$ John Smyth, ${ }^{1,2}$ Srinivas Bolisetty, ${ }^{1,2}$ Joanna Michalowski, ${ }^{1}$ Kei Lui ${ }^{1,2}$

To cite: Schindler T, Smyth J, Bolisetty S, et al. Early PARacetamol (EPAR) trial: a study protocol for a randomised controlled trial of early paracetamol to promote closure of the ductus arteriosus in preterm infants. BMJ Open 2019;9:e031428. doi:10.1136/ bmjopen-2019-031428

- Prepublication history and additional material for this paper are available online. To view these files, please visit the journal online (http://dx.doi. org/10.1136/bmjopen-2019031428).

Received 03 May 2019 Revised 02 August 2019 Accepted 30 August 2019

A) Check for updates

(C) Author(s) (or their employer(s)) 2019. Re-use permitted under CC BY-NC. No commercial re-use. See rights and permissions. Published by BMJ.

${ }^{1}$ Newborn Care, Royal Hospital for Women, Randwick, New South Wales, Australia

${ }^{2}$ School of Women's and Children's Health, University of New South Wales, Sydney, New South Wales, Australia

Correspondence to Dr Tim Schindler; tim.schindler@health.nsw. gov.au

\section{ABSTRACT}

Introduction The optimal management of patent ductus arteriosus (PDA) remains contentious. The medications used to treat PDA are often non-steroidal anti-inflammatory drugs, which are associated with a number of unwanted adverse effects. Paracetamol is a medication with an excellent safety profile in infants and has been suggested as a safe alternative medication in situations where other medications have failed or are contraindicated. There are limited data on the use of early, intravenous paracetamol in preterm infants.

Methods and analysis This trial aims to address whether early treatment with paracetamol will reduce the number of infants requiring intervention for PDA.

This is a randomised, double-blind, placebo-controlled trial in preterm infants $<29$ weeks' gestation. At 6 hours of life, infants with a ductus arteriosus $>0.9 \mathrm{~mm}$ will be randomised to receive either (1) intravenous paracetamol at a dose of $15 \mathrm{mg} / \mathrm{kg}$ initially, followed by every 6 hours at a dose of $7.5 \mathrm{mg} / \mathrm{kg}$ for 5 days; or (2) intravenous $5 \%$ dextrose every 6 hours for 5 days. The primary outcome is the need for any intervention for management of PDA up to 5 days. Secondary outcomes include closure of the ductus arteriosus at 5 days, size of the ductus arteriosus, ductal reopening, systemic blood flow, mortality and significant morbidities. The target sample size of 100 infants yields $>80 \%$ power, at the two-sided $5 \%$ level significance, to detect a $50 \%$ reduction in the need for intervention assuming that approximately $60 \%$ of infants in this study would otherwise have required intervention for PDA.

Ethics and dissemination A report on the results of the planned analyses will be prepared. The results of the primary analysis of all end points will be presented at medical conferences and submitted for publication in peer-reviewed journals. Separate manuscripts pertaining to the second aim of the study may be written, and these will also be submitted for publication in peerreviewed journals.

Trial registration number ACTRN12616001517460.

\section{Strengths and limitations of this study}

- This is a well-powered, blinded, randomised controlled trial to determine if early paracetamol will reduce the number of infants requiring intervention for patent ductus arteriosus (PDA).

- The primary outcome is an important clinical outcome, which is meaningful in everyday practice in the neonatal intensive care environment.

- Important pharmacological and safety data pertaining to the use of early paracetamol in preterm infants will be collected.

- The natural history of PDA would suggest that the ductus arteriosus will spontaneously close in a significant proportion of these infants, a recognised limitation of any study investigating interventions for PDA.

\section{INTRODUCTION}

\section{Background}

The ductus arteriosus is a fetal blood vessel that provides a communication between the pulmonary artery and the aorta, allowing oxygenated blood returning from the placenta to be diverted away from the fetal lung into the systemic circulation. As the newborn infant transitions from fetal to neonatal life, this blood vessel will usually close spontaneously within the first 72 hours of life. In preterm infants, it is very common for this physiological process to be delayed and the ductus arteriosus remains patent (PDA). Part of the process of circulatory adaptation at birth involves the pressure in the pulmonary circulation falling, allowing blood to flow to the lungs for oxygenation. A PDA potentially allows blood to flow left to right from the systemic circulation into the pulmonary circulation. This may result in excessive blood flow to the lungs, requiring 
increased respiratory support and may also result in poor blood flow to the body, providing inadequate blood flow to vital organs. More than half of preterm infants $<29$ weeks will require some form of intervention for a PDA, with four out of five infants born at 24 weeks requiring intervention. ${ }^{1}$

The optimal management of PDA is highly controversial with a lack of consensus regarding the need to treat, timing of intervention, the most appropriate pharmacological agents (including dose, dose intervals, duration, repeat courses, routes of administration) and the role of surgical intervention. Traditionally, the medications we use to treat PDA are non-steroidal anti-inflammatory drugs (NSAIDs), which decrease prostaglandin production by inhibiting cyclooxygenases (COX). This has a sound theoretical basis as persistently high prostaglandin levels result in vasodilatation and smooth muscle relaxation, which contributes to ductal patency. ${ }^{2}$ The most commonly used medications, indomethacin and ibuprofen, have a success rate of approximately $70 \%-85 \% .{ }^{3}{ }^{4}$ Unfortunately, these medications are associated with a number of unwanted adverse effects due to decreased blood flow to the brain, gastrointestinal tract and kidneys. Exposure to these medications puts vulnerable preterm infants at risk of significant complications such as intestinal perforation and necrotising enterocolitis. The alternative to medications is surgical intervention, which also carries significant risks, particularly for extreme preterm infants.

\section{Rationale}

Paracetamol is a medication with an excellent safety profile in infants when used to treat mild to moderate pain and fever. Although the mechanism of action of paracetamol is not completely understood, part of its spectrum of activity resembles that of a COX-2 selective inhibitor. Similar to traditional NSAIDs, this results in decreased prostaglandin production. It is therefore intuitive that this may also be effective in promoting ductal closure without the adverse effects associated with NSAIDs.

Early experiences with the use of paracetamol for ductal closure have been encouraging. The use of paracetamol in the preterm infant for PDA stems from an infant who was given paracetamol for other reasons. The treating team incidentally noted that the infant's PDA, which had previously been unresponsive to medical intervention, closed after administration of paracetamol. They then published their findings on a small case series of babies with resistant PDAs, all of whom responded to paracetamol. ${ }^{5}$ Twelve case reports and two randomised controlled trials have since explored the use of paracetamol in the management of PDA. ${ }^{6-18}$ Paracetamol appears to have similar efficacy to NSAIDs, without the gastrointestinal complications associated with NSAIDs, and is well tolerated in the preterm infant population. It has been suggested as a safe alternative medication in situations where other medications have failed or are contraindicated. ${ }^{19}$

The early haemodynamic impact of a large ductus arteriosus is evidenced by improvements in short-term outcomes seen when NSAIDs are used using an early prophylactic or targeted approach to treatment of the ductus arteriosus. ${ }^{3}{ }^{4} 20$ Although there is reasonable evidence of no harm with prophylactic treatment, there is an empirical logic in not exposing every infant to any medication, particularly in cases where the ductus arteriosus is either closed or was going to close spontaneously. Further to this, there is a growing interest in a conservative approach to management. ${ }^{21}$ Many neonatologists will therefore attempt to target treatment at those most likely to benefit. If paracetamol were to have similar efficacy to NSAIDs without the risk of adverse events, it may be possible to improve outcomes for our most vulnerable infants by giving early paracetamol to extreme preterm infants with a PDA.

\section{Evidence summary}

One published randomised controlled trial has compared paracetamol with placebo. ${ }^{22}$ In 48 preterm infants, intravenous paracetamol $(20 \mathrm{mg} / \mathrm{kg}$ loading; $7.5 \mathrm{mg} / \mathrm{kg}$ every 6 hours) was compared with no paracetamol within the first 24 hours of life, with a higher closure rate and shorter time to closure in the treatment arm and no reported adverse events. A systematic review of two randomised controlled trials reported comparisons of oral paracetamol versus oral ibuprofen for the treatment of an echocardiographically diagnosed PDA in 250 infants born preterm ( $\leq 34$ weeks postmenstrual age). The success rate for paracetamol to close a PDA was similar to that of ibuprofen. Adverse events were similar in both groups. However, in general the trends favoured infants who received paracetamol and additionally the adverse events were lower in the paracetamol group. ${ }^{23}$ A second systematic review of studies involving the use of paracetamol in preterm infants reported on 16 studies: two randomised controlled trials and 14 uncontrolled studies. The quality of selected studies was rated as poor. The majority of studies use $15 \mathrm{mg} / \mathrm{kg}$ every 6 hours for 3-7 days. Proportion meta-analysis of uncontrolled studies demonstrated a pooled ductal closure rate of $49 \%$ (95\% CI $29 \%$ to $69 \%$ ) and $76 \%$ (95\% CI $61 \%$ to $88 \%$ ) after 3 and 6 days of treatment with paracetamol, respectively. ${ }^{24}$

\section{Aim}

1. To study the effect of early treatment of PDA with paracetamol.

2. To examine the safety and efficacy profile of paracetamol during the early postnatal period.

\section{Hypotheses}

We hypothesise that:

1. Early treatment with paracetamol will reduce the number of infants requiring intervention for PDA.

2. The use of paracetamol in preterm infants with a PDA will result in a higher rate of ductal closure compared with placebo.

3. Paracetamol can be used safely in preterm infants during the early postnatal period. 
Table 1 Trial synopsis

\begin{tabular}{ll} 
Study title & Early PARacetamol to promote early closure of the ductus arteriosus \\
\hline Study aims & $\begin{array}{l}\text { 1. To study the effect of early treatment of patent ductus arteriosus with paracetamol } \\
\text { 2. To examine the safety and efficacy profile of paracetamol during the early postnatal period }\end{array}$ \\
Outcomes & $\begin{array}{l}\text { Primary: any intervention for management of PDA up to } 5 \text { days } \\
\text { Secondary: closure of ductus arteriosus at } 5 \text { days; size of ductus arteriosus at } 48 \text { hours and } 5 \text { days; ductal reopening } \\
\text { during admission; ductus arteriosus parameters; systemic blood flow measurements; adverse events during the treatment } \\
\text { period; mortality; significant morbidities }\end{array}$ \\
Design & $\begin{array}{l}\text { Double-blind, placebo-controlled, parallel, two-arm, randomised, phase II single-centre trial, stratified by gestational age } \\
\text { and size of ductus at initial assessment }\end{array}$ \\
Inclusion criteria & $\begin{array}{l}\text { 1. Babies }<6 \text { hours old } \\
\text { 2. Born }<29 \text { weeks' gestation }\end{array}$ \\
3. Ductus arteriosus $\geq 1.0 \mathrm{~mm} ;<30 \% \mathrm{R}-\mathrm{L}$ shunt \\
4. Informed parental consent
\end{tabular}

Exclusion criteria 1 . Known congenital anomalies
2. Haemodynamic instability ( $>1$ ionotropic agent)
3. Abnormal baseline liver function
4. Clinician decision to give indomethacin prophylaxis
5. Ductus arteriosus $<1.0 \mathrm{~mm} ; \geq 30 \% \mathrm{R}-\mathrm{L}$ shunt

Intervention Intervention: intravenous paracetamol $15 \mathrm{mg} / \mathrm{kg}$ loading; $7.5 \mathrm{mg} / \mathrm{kg}$ maintenance Control: intravenous $5 \%$ dextrose $1.5 \mathrm{~mL} / \mathrm{kg}$ loading; $0.75 \mathrm{~mL} / \mathrm{kg}$ maintenance

Study product Active: paracetamol $10 \mathrm{mg} / \mathrm{mL}$ Placebo: $5 \%$ dextrose

Treatment schedule Loading dose to be given at 6 hours after clinician performed ultrasound; maintenance doses to be given every 6 hours for total 5 days

Routine assessments to be performed at 48 hours and 5 days

The treating team will manage infants enrolled in the study as they would manage any other infant. If there is a clinical decision that a PDA requires intervention, based on either a routine assessment or otherwise, the trial intervention will be discontinued. Intervention and ongoing management will then be at the discretion of the treating neonatologist

$\begin{array}{ll}\text { Preparation } & \begin{array}{l}\text { Both paracetamol and } 5 \% \text { dextrose are clear, colourless and indistinguishable. } 1.5 \mathrm{~mL} / \mathrm{kg} \text { (loading) or } 0.75 \mathrm{~mL} / \mathrm{kg} \\ \text { (maintenance) of active treatment or placebo will be prepared and administered as per hospital protocol for paracetamol }\end{array} \\ \text { Blood samples } & \begin{array}{l}\text { Liver function tests to be added routine blood tests; paracetamol levels to be added to blood tests on day } 2 \text { and day } 5 \text {; all } \\ \text { blood tests should be add-ons, no additional blood samples are required }\end{array} \\ \text { Sample size } & \begin{array}{l}\mathrm{n}=100 ; 50 / \text { group; assuming that approximately } 60 \% \text { of infants in this study would otherwise have required intervention } \\ \text { for PDA, a sample size of at least } 42 \text { infants per group would be required to detect a } 50 \% \text { reduction in the need for } \\ \text { intervention, with } 95 \% \mathrm{Cl} \text { and power of } 80 \%\end{array}\end{array}$

PDA, patent ductus arteriosus.

\section{METHODS AND ANALYSIS}

\section{Trial design}

Double-blind, placebo-controlled, parallel, two-arm, randomised, phase II single-centre trial, stratified by gestational age and size of ductus at initial assessment. A trial synopsis (table 1) and flowchart (figure 1) summarise the trial design.

\section{Eligibility criteria}

Preterm infants will be screened for eligibility on admission to the newborn intensive care unit. To be eligible for the study, infants must meet the following inclusion criteria: infants born at $<29$ weeks' gestation; less than 6 hours old; ductus arteriosus $\geq 1.0 \mathrm{~mm}$ with $<30 \%$ right to left shunt. Exclusion criteria are known congenital anomalies, haemodynamic instability ( $>1$ ionotropic agent), abnormal baseline liver function (transaminases $>50 \%$ above upper reference range or bilirubin above local guideline for exchange transfusion), clinician decision to give indomethacin prophylaxis, and ductus arteriosus
$<1.0 \mathrm{~mm}$ or $\geq 30 \%$ right to left shunt. Infants of multiple births are eligible and will be randomised individually.

\section{Initial assessment}

Eligible infants will be assessed at 6 hours of life after obtaining parental consent. A clinician performed cranial and cardiac ultrasound will be attended by the medical team. All images will be reviewed by a neonatologist credentialled with a Certificate in Clinician Performed Ultrasound. At this time point, and at each subsequent assessment point, a number of ultrasound parameters will be documented, summarised in table 2 .

\section{Intervention}

Infants who meet all inclusion criteria and who have a demonstrable ductus arteriosus that is shunting predominantly left to right (implying normal circulatory adaptation) will be randomised to receive either (1) intravenous paracetamol at a dose of $15 \mathrm{mg} / \mathrm{kg}$ initially, followed by every 6 hours at a dose of $7.5 \mathrm{mg} / \mathrm{kg}$ for 5 days; or (2) intravenous $5 \%$ dextrose every 6 hours for 5 days. Intravenous 


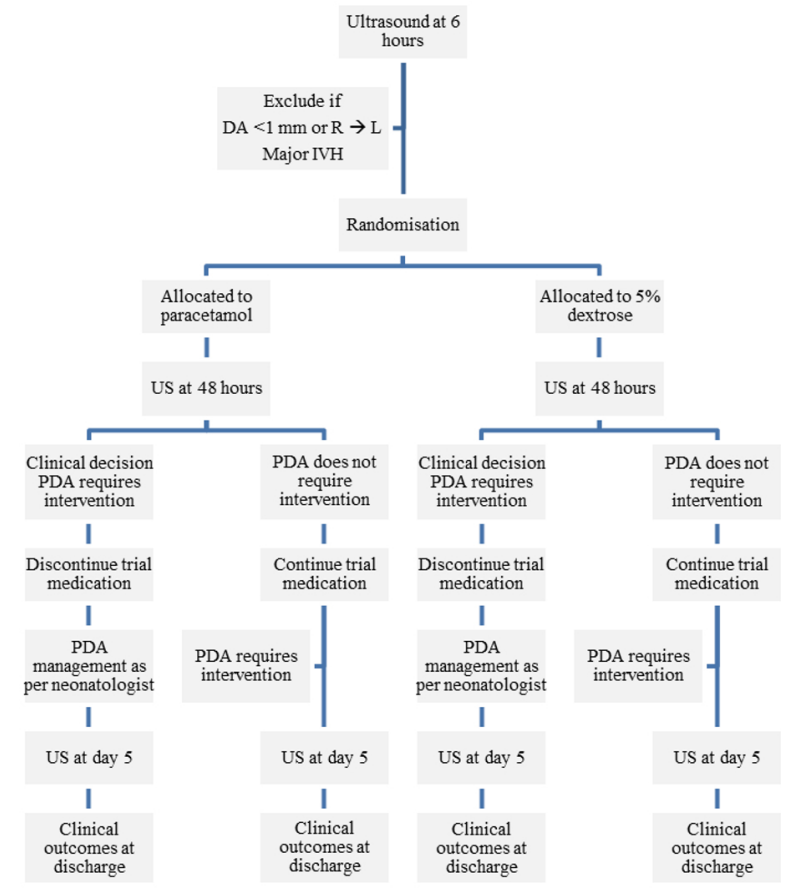

Figure 1 Trial flowchart. IVH, intraventricular haemorrhage; PDA, patent ductus arteriosus.

paracetamol and 5\% dextrose will be prepared in identical syringes. Both preparations are clear, colourless and have no identifiable differences. All doses of trial medication will be prepared and checked by the nursing team leader and a registered nurse who is not looking after the infant.

\section{Paracetamol dosing}

The decision on the paracetamol dose regimen required careful consideration as the data regarding pharmacokinetics, pharmacodynamics and toxicity of paracetamol in extremely preterm infants is limited. Dose regimes of $15 \mathrm{mg} / \mathrm{kg}$ every 6 hours have been used in most published data investigating treatment of PDA using intravenous paracetamol. ${ }^{12-16}$ However, the concern with regular high-dose paracetamol is the potential for hepatotoxicity due to accumulation of toxic metabolites, particularly in

\begin{tabular}{|c|c|}
\hline Cardiac US & Head US \\
\hline $\begin{array}{l}\text { Ductus arteriosus parameters } \\
\text { Size, flow pattern } \\
\text { Diastolic flow in descending } \\
\text { aorta } \\
\text { (retrograde/absent/antegrade) } \\
\text { Left pulmonary artery diastolic } \\
\text { velocity }\end{array}$ & $\begin{array}{l}\text { Intraventricular } \\
\text { haemorrhage } \\
\text { Presence or absence } \\
\text { Grade if present }\end{array}$ \\
\hline $\begin{array}{l}\text { Systemic blood flow measurements } \\
\text { Left ventricular output } \\
\text { Right ventricular output } \\
\text { Superior vena cava blood flow }\end{array}$ & $\begin{array}{l}\text { Other pathology } \\
\text { Other intracranial } \\
\text { bleeding } \\
\text { Periventricular } \\
\text { leukomalacia }\end{array}$ \\
\hline
\end{tabular}

the immediate neonatal period when paracetamol clearance may be low. Based on pharmacokinetic prediction modelling, it is likely that a modest loading dose followed by a more conservative regular dose will quickly achieve a good median paracetamol concentration at levels that will optimise safety. ${ }^{25}$ It is important to acknowledge, however, that the target concentration for ductal closure is unknown. The selected paracetamol regimen was based on a combination of doses used in previous studies and pharmacokinetic prediction modelling. Paracetamol concentrations are predicted to be $13 \mathrm{mg} / \mathrm{L}, 11 \mathrm{mg} / \mathrm{L}$ and $10 \mathrm{mg} / \mathrm{L}$ for infants weighing $0.5 \mathrm{~kg}, 1.0 \mathrm{~kg}$ and $1.5 \mathrm{~kg}$, respectively. ${ }^{25}$

\section{Randomisation}

Randomisation will be achieved by using randomly generated treatment allocations within sealed opaque envelopes. Randomisation will be stratified based on gestational age (23-25 weeks; 26-28 weeks) and size of ductus arteriosus $(\leq 1.5 \mathrm{~mm} ;>1.5 \mathrm{~mm})$ at initial assessment. All clinical staff looking after the infants will be blinded as to the study group assignment. Infants with major intraventricular haemorrhage will be excluded before randomisation.

\section{Unblinding}

Blinding of families to treatment allocation will be maintained throughout the trial. Unblinding is not generally necessary for the management of a participant with an adverse event and this is strongly discouraged. If the treating team felt that unblinding were necessary, they are encouraged to discuss this with one of the principal investigators.

\section{Routine assessments}

All infants will be routinely assessed at 48 hours of life with cranial and cardiac ultrasound. These results will be communicated with the treating team who will decide whether any PDA requires intervention. The trial intervention will be discontinued if there is evidence of significant pulmonary hypertension (defined by either suprasystemic pulmonary pressures or $>30 \%$ right to left ductal shunt). The treatment course will be completed in cases where the ductus arteriosus appears to be closing or is closed. A final routine assessment will performed at the end of the treatment course ( 5 days). Additional interim assessments may be performed as clinically indicated. At all times, infants will remain under the care of the treating neonatologist. Clinical decisions, including PDA management, will always be at the discretion of the treating team. Ongoing management of the PDA after 5 days will continue as per the treating team.

As a guide for the treating team, the following criteria should be met before considering rescue treatment:

- The infant should have a clinically apparent PDA on the basis of physical signs such as murmur, active praecordium or full pulses. 
- The infant should have clinical features that suggest the PDA is having a clinical impact such as ionotroperesistant hypotension, pulmonary haemorrhage, ventilator dependence, respiratory instability including increasing apnoea and rising oxygen requirements or feed intolerance (unable to tolerate minimal enteric feeds).

- Ultrasound findings should confirm patency with significant shunt defined by:

- Diameter at narrowest point of more than or equal to $2.0 \mathrm{~mm}$ with one or more of the following:

- Reversed diastolic flow in the postductal descending aorta.

- Increased velocity in the left pulmonary artery (diastolic $>0.2 \mathrm{~m} / \mathrm{s}$, mean $>0.45 \mathrm{~m} / \mathrm{s}$ ).

- Dilation of the left atrium and/or left ventricle $(>1.4: 1)$.

\section{Data collection}

The following variables will be recorded for infants included in the study: demographic data, gestational age, birth weight, birth weight percentile, sex, mode of delivery, Apgar score at 5 min, plurality, exposure to antenatal steroids, maternal history of hypertensive disease of pregnancy, antepartum haemorrhage or chorioamnionitis. Clinical outcomes will be assessed at discharge.

\section{Outcomes}

Primary

1. Any intervention for management of PDA up to 5 days.

\section{Secondary}

1. Closure of ductus arteriosus at 5 days.

2. Size of ductus arteriosus at 48 hours and 5 days.

3. Ductal reopening during admission.

4. Ductus arteriosus parameters.

5. Systemic blood flow measurements.

6. Adverse events during the treatment period.

7. Clinical outcomes including mortality and significant morbidities (pulmonary haemorrhage, necrotising enterocolitis, early-onset sepsis, late-onset sepsis, intraventricular haemorrhage, periventricular leukomalacia, chronic lung disease, retinopathy of prematurity, hepatic impairment, renal impairment).

\section{Outcome data definitions}

- Pulmonary haemorrhage: any blood suctioned from the trachea.

- Necrotising enterocolitis: necrotising enterocolitis proven either radiologically or at surgery ( $\geq$ stage II Bells).

- Early-onset sepsis: clinical picture consistent with sepsis within the first 48 hours of life and a positive bacterial or fungal culture of blood and/or cerebrospinal fluid.

- Late-onset sepsis: clinical picture consistent with sepsis after the first 48 hours of life and a positive bacterial or fungal culture of blood and/or cerebrospinal fluid.
- Intraventricular haemorrhage: worst grade of intraventricular haemorrhage using Papile Classification ${ }^{26}$ seen on either side of the head by imaging or postmortem examination.

- Chronic lung disease: any respiratory support at 36 or 40 weeks' corrected gestational age.

- Retinopathy of prematurity: the worst stage as described by the Committee for Classification of Retinopathy of Prematurity. ${ }^{27}$

- Hepatic impairment: transaminases $>50 \%$ above upper reference range.

- Renal impairment: creatinine $>50 \%$ above upper reference range.

\section{Rescue treatment and discontinuation of intervention}

The treating team will continue to manage infants enrolled in the study as they would treat any other infant. If there is a clinical decision that a PDA requires intervention, based on either the routine assessment at 48 hours of life or otherwise, the trial intervention will be discontinued. Intervention and ongoing management will then be at the discretion of the treating neonatologist.

\section{Other medications}

Participants in the trial may receive any medications required, either routine medications given in the context of prematurity or medications for comorbid conditions. Off-protocol use of paracetamol or any NSAIDs is not permitted while participants are still receiving the trial medication. If there is a clinical decision that a PDA requires intervention at any time point, this constitutes treatment failure and the treating neonatologist may use any medication that they deem appropriate, including paracetamol. Any medication known to cause liver dysfunction should be used with caution while participants are receiving the trial medication.

\section{Statistical analysis}

All tests of the effect of treatment on outcomes will be conducted on an intention-to-treat basis. That is, all randomised patients will be analysed in the group to which they were randomised regardless of whether they received the assigned treatment and regardless of any protocol deviations or violations. Analyses of outcome variables will, however, exclude data from infants who withdraw from study treatment and withdraw consent for use of their data. All primary statistical analyses will be unadjusted and tests of significance will be two sided. Any departures from intention to treat will be documented and reported.

\section{Sample size}

With regard to sample size calculation, we propose the following:

Assuming that approximately $60 \%$ of infants in this study would otherwise have required intervention for PDA, ${ }^{1}$ a sample size of at least 42 infants per group would be required to detect a $50 \%$ reduction in the need for intervention, with a $95 \% \mathrm{CI}$ and a power of $80 \%$. 


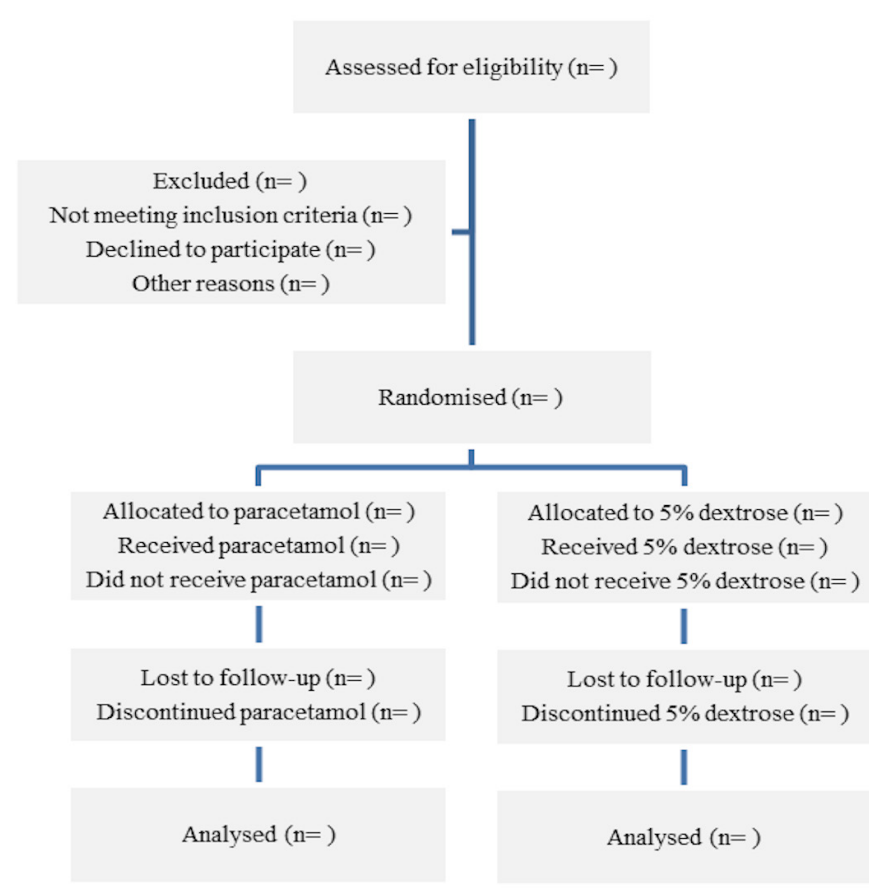

Figure 2 Flowchart of progression through the trial.

Calculation based on the following ${ }^{28}$ :

$$
N=\frac{2\left[z_{\alpha / 2} \sqrt{2 \bar{p} q}+z_{\beta} \sqrt{p_{0} q_{0}+p_{1} q_{1}}\right]^{2}}{\left(p_{0}-p_{1}\right)^{2}}
$$

Trial profile

A CONSORT-style flow diagram will illustrate infant progression through the trial from initial screening for eligibility to completion of the primary outcome assessment (see figure 2).

\section{Infant characteristics and baseline comparisons}

Demographic and other baseline characteristics will be summarised by assigned treatment group. Categorical variables will be summarised by frequencies and percentages. Percentages will be calculated according to the number of patients for whom data are available. Where values are missing, the denominator will be reported. Continuous variables will be summarised by mean and $\mathrm{SD}$ as well as by quartiles. Differences between groups will be determined by $\chi^{2}$ or Fisher's exact test for categorical data, t-test for parametric continuous data and Wilcoxon rank-sum test for non-parametric continuous data.

Analysis of the primary outcome

Any intervention for management of PDA up to 5 days

The need for medical or surgical intervention will be assessed as a binary outcome for each infant. Data will be analysed by treatment group using a $\chi^{2}$ or Fisher's exact test as appropriate.

Analysis of secondary outcomes

Closure of ductus arteriosus at 5 days

Closure of the ductus arteriosus will be assessed as a binary outcome for each infant. Data will be analysed by treatment group using a $\chi^{2}$ or Fisher's exact test as appropriate.

\section{Size of ductus arteriosus at 48 hours and 5 days}

The size of the ductus arteriosus will be measured in millimetres. Means and SD or medians and IQRs will be reported, as appropriate. A t-test or Wilcoxon rank-sum test will be used depending on the distribution of data. The flow pattern will be categorised into one of bidirectional, growing, pulsatile, closing or closed. Differences between groups will be determined by $\chi^{2}$ or Fisher's exact test as appropriate.

\section{Ductal reopening during admission}

Ductal reopening will be assessed as a binary outcome for each infant. Data will be analysed by treatment group using a $\chi^{2}$ or Fisher's exact test as appropriate.

\section{Ductus arteriosus parameters (excluding size and pattern)}

Diastolic flow in the descending aorta will be categorised into one of retrograde, absent or antegrade. Differences between groups will be determined by $\chi^{2}$ or Fisher's exact test as appropriate. Left pulmonary artery diastolic velocity will be measured in metres per second. Means and SD or medians and IQRs will be reported as appropriate. A t-test or Wilcoxon rank-sum test will be used depending on the distribution of data.

\section{Systemic blood flow measurements}

Measures of systemic blood flow will be measured in millilitres per kilogram per minute. Means and SD or medians and IQRs will be reported, as appropriate. A t-test or Wilcoxon rank-sum test will be used depending on the distribution of data.

\section{Clinical outcomes including mortality and significant morbidities}

Clinical outcomes will be defined as present or absent for each infant. Each binary clinical outcome will be analysed by treatment group using a $\chi^{2}$ or Fisher's exact test as appropriate.

\section{Analysis of safety outcomes}

Safety outcomes will be defined as present or absent for each infant. Each binary safety outcome will be analysed by treatment group using a $\chi^{2}$ or Fisher's exact test as appropriate.

\section{Subgroup analysis}

The following subgroup analyses are pre-specified:

- Size of ductus arteriosus compared with median at initial assessment $(\leq 1.5 \mathrm{~mm} ;>1.5 \mathrm{~mm})$.

- Gestational age groups (23-25 weeks; 26-28 weeks).

Interim analysis

An interim analysis and review of the study by the data and safety monitoring board will be performed at $50 \%$ 
recruitment. The criteria for stopping the trial at this point are as follows:

- Reported serious adverse events (SAEs) in the treatment arm using Haybittle-Peto rule of 3 SDs $(2 \mathrm{p}=0.003)$.

- Stopping for benefit will be based on Haybittle-Peto rule of 3 SDs $(2 p=0.003)$.

- Stopping for harm will use a two-sided alpha of 0.01.

\section{Patient and public involvement}

Running for Premature Babies is a registered charity that supports the research needs of the Royal Hospital for Women's Neonatal Intensive Care Unit. The Running for Premature Babies group is supporting this study and was engaged at study conception through the charity founder, ex-parent Sophie Smith. The research question and trial proposal were discussed in detail, including how participation in the study would impact on infants enrolled in the trial. Progress reports have been and will continue to be provided to this group at regular intervals.

The Royal Hospital for Women's Quality and Patient Safety Committee, which includes consumer representation, has also been involved since study conception. The committee reviewed the study and approved the concept and design of the study, which included the trial's acceptability to participating infants and their families. The Royal Hospital for Women Foundation, a fundraising team dedicated to the Royal Hospital for Women, has also been engaged and supports the conduct of this study.

The results of this study will be made available to any participating family by request. A lay summary of the results will be prepared specifically for this purpose. Running for Premature Babies and the Royal Hospital for Women have both provided their members and supporters study information in the form of lay information on their respective websites, emails and newsletters. Both organisations will be invited to similarly disseminate the results of the study when available at the conclusion of the study.

\section{ETHICS AND DISSEMINATION \\ Ethics}

This study is conducted according to the Note for guidance on Good Clinical Practice (CPMP/ICH/135/96) annotated with TGA comments DSEB, July 2000, section 4 and the NHMRC National Statement on Ethical Conduct in Human Research.

\section{Consent}

Parents of eligible infants are informed of the purpose of the study, what is involved, the possible risks of study participation, the voluntary nature of participation, the right to withdraw and protection of confidentiality by the investigators. The parents are provided with a written information sheet (see online e-supplemental 1), and evidence of informed consent is obtained in writing.

\section{Safety}

\section{Monitoring}

Daily liver function tests will be used to monitor infants for evidence of hepatotoxicity. The trial intervention will be discontinued in the event of liver dysfunction (transaminases $>50 \%$ above upper reference range or bilirubin above local guideline for exchange transfusion). Paracetamol levels will also be checked on day 2 and day 5 to investigate the pharmokinetics of paracetamol in this population. These results will not be made available to clinical staff or study personnel. In the event of a paracetamol level $>500 \mu \mathrm{mol} / \mathrm{L}(75 \mathrm{mg} / \mathrm{L})$, the trial management committee will be notified immediately and the trial intervention will be discontinued.

\section{Trial discontinuation}

The trial intervention will be discontinued in the event of any significant adverse events including intestinal perforation, necrotising enterocolitis, suspected sepsis, major intraventricular haemorrhage and periventricular leukomalacia. All events that result in trial discontinuation will be reported immediately to the trial management committee.

\section{Data and safety monitoring board}

A data and safety monitoring board (DSMB) will monitor the progress of the study following a specific charter (see online e-supplemental 2). The trial management committee is responsible for reporting all SAEs, including suspected unexpected serious adverse reactions, to the DSMB.

\section{Data management}

The study will be conducted in accordance with applicable Privacy Acts and Regulations. All information will be stored securely at the Department of Newborn Care, Royal Hospital for Women. It will only be available to staff directly involved with the study. Access to anonymised data, or other study materials, by other parties will be at the discretion of the trial management committee. All study-related documentation will be maintained for 7 years following completion of the trial.

Contributors TS conceived the study. TS, JS, SB and KL designed the study. TS and JM co-ordinated the implementation of the study. TS and JS are responsible for image acquisition. TS drafted the manuscript. All authors (TS, JS, SB, JM and KL) reviewed and revised the manuscript, providing important intellectual content, and approved the final version.

Funding This work was supported by Running for Premature Babies, a fund raising group that provides substantial research support to the Department of Newborn Care at the Royal Hospital for Women.

Competing interests None declared.

Patient consent for publication Not required.

Ethics approval Ethical approval was granted by the South Eastern Sydney Local Health District Human Research Ethics Committee in September 2015 (current protocol-V.6, 13 February 2019)

Provenance and peer review Not commissioned; externally peer reviewed.

Open access This is an open access article distributed in accordance with the Creative Commons Attribution Non Commercial (CC BY-NC 4.0) license, which permits others to distribute, remix, adapt, build upon this work non-commercially, 
and license their derivative works on different terms, provided the original work is properly cited, appropriate credit is given, any changes made indicated, and the use is non-commercial. See: http://creativecommons.org/licenses/by-nc/4.0/.

\section{REFERENCES}

1 Bolisetty S, Legge N, Bajuk B, et al. Preterm infant outcomes in New South Wales and the Australian Capital Territory. J Paediatr Child Health 2015;51:713-21.

2 Hermes-DeSantis ER, Clyman RI. Patent ductus arteriosus: pathophysiology and management. J Perinatol 2006;26:S14-18.

3 Fowlie PW, Davis PG, McGuire W, et al. Prophylactic intravenous indomethacin for preventing mortality and morbidity in preterm infants. Cochrane Database Syst Rev 2010;115.

4 Ohlsson A, Shah SS, Cochrane Neonatal Group. Ibuprofen for the prevention of patent ductus arteriosus in preterm and/or low birth weight infants. Cochrane Database Syst Rev 2011;89.

5 Hammerman C, Bin-Nun A, Markovitch E, et al. Ductal closure with paracetamol: a surprising new approach to patent ductus arteriosus treatment. Pediatrics 2011;128:e1618-21.

6 Oncel MY, Yurttutan S, Uras N, et al. An alternative drug (paracetamol) in the management of patent ductus arteriosus in ibuprofen-resistant or contraindicated preterm infants. Arch Dis Child Fetal Neonatal Ed 2013;98:F94.

7 Jasani B, Kabra N, Nanavati RN. Oral paracetamol in treatment of closure of patent ductus arteriosus in preterm neonates. J Postgrad Med 2013:59:312-4.

8 Özdemir Özmert M. A., Doğan M, Küçüktașçı K, et al. Paracetamol therapy for patent ductus arteriosus in premature infants: a chance before surgical ligation. Pediatr Cardiol 2014;35:276-9.

9 Sinha R, Dalal SS, Negi V. An interesting observation of PDA closure with oral paracetamol in preterm neonates. J Clin Neonatol 2013;2:30-2.

10 Kessel I, Waisman D, Lavie-Nevo K, et al. Paracetamol effectiveness, safety and blood level monitoring during patent ductus arteriosus closure: a case series. J Matern Fetal Neonatal Med 2014;27:1719-21.

11 Nadir E, Kassem E, Foldi S, et al. Paracetamol treatment of patent ductus arteriosus in preterm infants. J Perinatol 2014;34:748-9.

12 Oncel MY, Yurttutan S, Degirmencioglu H, et al. Intravenous paracetamol treatment in the management of patent ductus arteriosus in extremely low birth weight infants. Neonatology 2013;103:166-9.

13 Alan S, Kahvecioglu D, Erdeve O, et al. Is paracetamol a useful treatment for ibuprofen-resistant patent ductus arteriosus? Neonatology 2013;104:168-9.
14 Roofthooft DWE, van Beynum IM, Helbing WA, et al. Paracetamol for ductus arteriosus closure: not always a success story. Neonatology 2013;104:170.

15 Tekgunduz KS, Ceviz N, Demirelli Y, et al. Intravenous paracetamol for patent ductus arteriosus in premature infants - a lower dose is also effective. Neonatology 2013;104:6-7.

16 Terrin G, Conte F, Scipione A, et al. Efficacy of paracetamol for the treatment of patent ductus arteriosus in preterm neonates. Ital $\mathrm{J}$ Pediatr 2014;40:21.

17 Oncel MY, Yurttutan S, Erdeve O, et al. Oral paracetamol versus oral ibuprofen in the management of patent ductus arteriosus in preterm infants: a randomized controlled trial. J Pediatr 2014;164:510-4.

18 Dang D, Wang D, Zhang C, et al. Comparison of oral paracetamol versus ibuprofen in premature infants with patent ductus arteriosus: a randomized controlled trial. PLoS One 2013;8:e77888.

19 Le J, Gales MA, Gales BJ. Acetaminophen for patent ductus arteriosus. Ann Pharmacother 2015;49:241-6.

20 Kluckow M, Jeffery M, Gill A, et al. A randomised placebo-controlled trial of early treatment of the patent ductus arteriosus. Arch Dis Child Fetal Neonatal Ed 2014;99:F99-104.

21 Hundscheid T, Onland W, van Overmeire B, et al. Early treatment versus expectative management of patent ductus arteriosus in preterm infants: a multicentre, randomised, non-inferiority trial in Europe (BeNeDuctus trial). BMC Pediatr 2018;18:262.

22 Härkin P, Härmä A, Aikio O, et al. Paracetamol accelerates closure of the ductus arteriosus after premature birth: a randomized trial. $J$ Pediatr 2016;177:72-7.

23 Ohlsson A, Shah PS, Paracetamol SPS, Cochrane Neonatal Group. Paracetamol (acetaminophen) for patent ductus arteriosus in preterm or low-birth-weight infants. Cochrane Database Syst Rev 2015;8.

24 Terrin G, Conte F, Oncel MY, et al. Paracetamol for the treatment of patent ductus arteriosus in preterm neonates: a systematic review and meta-analysis. Arch Dis Child Fetal Neonatal Ed 2016;101:F127-36.

25 Wang C, Allegaert K, Tibboel D, et al. Population pharmacokinetics of paracetamol across the human age-range from (pre)term neonates, infants, children to adults. J Clin Pharmacol 2014;54:619-29.

26 Papile L-A, Burstein J, Burstein R, et al. Incidence and evolution of subependymal and intraventricular hemorrhage: a study of infants with birth weights less than 1,500 GM. J Pediatr 1978;92:529-34.

27 International Committee for the Classification of Retinopathy of Prematurity. The International classification of retinopathy of prematurity revisited. Arch Ophthalmol 2005;123:991-9.

28 Bell ML, Teixeira-Pinto A, McKenzie JE, et al. A myriad of methods: calculated sample size for two proportions was dependent on the choice of sample size formula and software. J Clin Epidemiol 2014;67:601-5. 